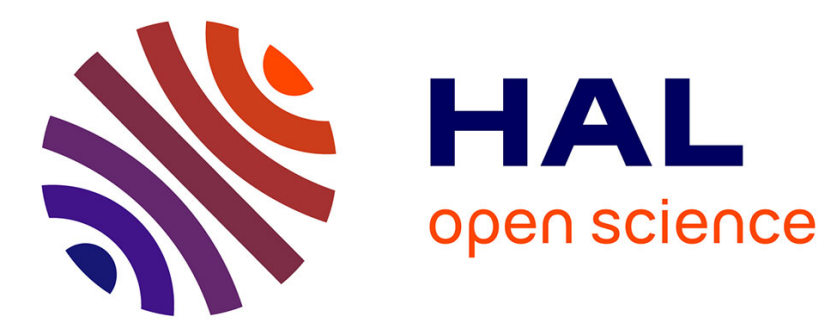

\title{
Kinks and d-waves from phonons: The intermediate coupling story
}

\author{
J.P. Hague
}

\section{To cite this version:}

J.P. Hague. Kinks and d-waves from phonons: The intermediate coupling story. Journal of Physics and Chemistry of Solids, 2009, 10.1016/j.jpcs.2008.06.105 . hal-00587298

\section{HAL Id: hal-00587298 \\ https://hal.science/hal-00587298}

Submitted on 20 Apr 2011

HAL is a multi-disciplinary open access archive for the deposit and dissemination of scientific research documents, whether they are published or not. The documents may come from teaching and research institutions in France or abroad, or from public or private research centers.
L'archive ouverte pluridisciplinaire HAL, est destinée au dépôt et à la diffusion de documents scientifiques de niveau recherche, publiés ou non, émanant des établissements d'enseignement et de recherche français ou étrangers, des laboratoires publics ou privés. 


\section{Author's Accepted Manuscript}

Kinks and d-waves from phonons: The intermediate coupling story

J.P. Hague

PII:

S0022-3697(08)00220-5

DOI:

Reference:

doi:10.1016/j.jpcs.2008.06.105

PCS 5523

To appear in: $\quad$ Journal of Physics and

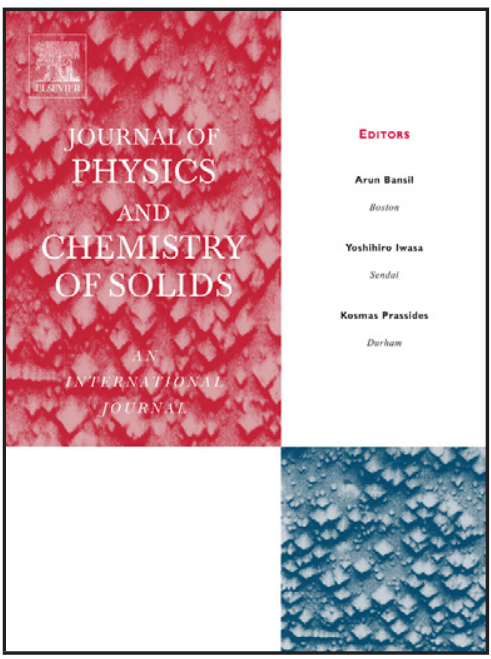

www.elsevier.com/locate/jpcs Chemistry of Solids

Cite this article as: J.P. Hague, Kinks and d-waves from phonons: The intermediate coupling story, Journal of Physics and Chemistry of Solids (2008), doi:10.1016/j.jpcs.2008.06.105

This is a PDF file of an unedited manuscript that has been accepted for publication. As a service to our customers we are providing this early version of the manuscript. The manuscript will undergo copyediting, typesetting, and review of the resulting galley proof before it is published in its final citable form. Please note that during the production process errors may be discovered which could affect the content, and all legal disclaimers that apply to the journal pertain. 


\title{
Kinks and d-waves from phonons: The intermediate coupling story
}

\author{
J.P.Hague ${ }^{a}$ \\ ${ }^{a}$ Department of Physics, Loughborough University, Loughborough, LE11 3TU, UK
}

\begin{abstract}
I present results from an approach that extends the Eliashberg theory by systematic expansion in the vertex function; an essential extension at large phonon frequencies, even for weak coupling. In order to deal with computationally expensive double sums over momenta, a dynamical cluster approximation (DCA) approach is used to incorporate momentum dependence into the Eliashberg equations. First, I consider the effects of introducing partial momentum dependence on the standard Eliashberg theory using a quasi-local approximation; which I use to demonstrate that it is essential to include corrections beyond the standard theory when investigating $d$-wave states. Using the extended theory with vertex corrections, I compute electron and phonon spectral functions. A kink in the electronic dispersion is found in the normal state along the major symmetry directions, similar to that found in photo-emission from cuprates. The phonon spectral function shows that for weak coupling $W \lambda<\omega_{0}$, the dispersion for phonons has weak momentum dependence, with consequences for the theory of optical phonon mediated d-wave superconductivity, which is shown to be 2 nd order in $\lambda$. In particular, examination of the order parameter vs. filling shows that vertex corrections lead to $d$-wave superconductivity mediated via simple optical phonons. I map out the order parameters in detail, showing that there is significant induced anisotropy in the superconducting pairing in quasi-2D systems.
\end{abstract}

Key words: Extended Eliashberg Theory, Superconductivity, Spectroscopy, Unconventional Pairing

\section{Introduction}

Angle-resolved photo-emission spectroscopy (ARPES) directly probes the dispersion of electrons; identifying a kink associated with the active optical phonon in the cuprates [1]. Neutron scattering has shown an anomalous change in the phonon spectrum at the superconducting transition, indicating an interesting role for phonons in the cuprates [2]. Estimates of the magnitude of the electron-phonon coupling, and the energy of the phonon mode put the problem outside the limited region of applicability for BCS theory, so schemes to cope with a wider range of parameters need to be developed. Moreover, since there a node in the superconduct-

\footnotetext{
* Tel: +44-1509-228208. Fax: +44-1509-223986

Email address: J.P.Hague@lboro.ac.uk (J.P.Hague)

1 I acknowledge support by EPSRC grant EP/C518365/1
}

ing gap consistent with d-wave symmetry [3], any theory implicating phonons as the mechanism must be able to explain the unconventional order.

Electron-phonon (e-ph) interactions can be described by the following generic model,

$$
\begin{aligned}
H=\sum_{\mathbf{k} \sigma} \epsilon_{\mathbf{k}} c_{\mathbf{k}, \sigma}^{\dagger} c_{\mathbf{k}, \sigma}+\sum_{k q} \frac{g_{\mathbf{q}}}{\sqrt{\omega_{\mathbf{q}}}} c_{\mathbf{k}-\mathbf{q}, \sigma}^{\dagger} c_{\mathbf{k}, \sigma}\left(b_{\mathbf{q}}^{\dagger}+b_{\mathbf{q}}\right) \\
+\sum_{\mathbf{q}} \omega_{\mathbf{q}}\left(b_{\mathbf{q}}^{\dagger} b_{\mathbf{q}}+1 / 2\right)
\end{aligned}
$$

Here, $\hbar=1$ and $\epsilon_{\mathbf{k}}=-2 t\left(\cos \left(a k_{x}\right)+\cos \left(a k_{y}\right)\right)-$ $2 t_{\perp} \cos \left(c k_{z}\right)$ with $t=0.25$ and $t_{\perp}=0.01$ (representing a quasi-2D system and taming the vanHove singularities). The Holstein model is a special case where $g_{\mathbf{q}}=g_{0}$ and $\omega_{\mathbf{q}}=\omega_{0}$, for which $\lambda=g_{0}^{2} /\left(\omega_{0}^{2} W\right)$ is the dimensionless e-ph coupling ( $W$ is the half-band width). The momentumindependent phonon dispersion approximates an 
optical phonon, and the momentum-independent e-ph coupling corresponds to a completely local interaction. A Coulomb pseudo-potential, $H_{\mu}=$ $-W \mu_{c} \sum_{\mathbf{k q} \sigma}\left\langle c_{\mathbf{q} \sigma}^{\dagger} c_{-\mathbf{q} \bar{\sigma}}^{\dagger}\right\rangle c_{\mathbf{k} \sigma} c_{-\mathbf{k} \bar{\sigma}}+h . c .$, may be added (i.e. Hubbard model at the Hartree-Fock level). (a)

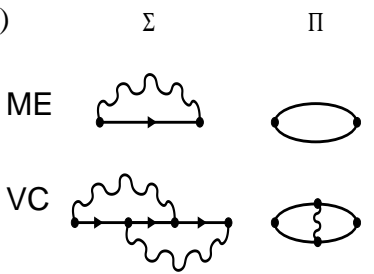

(b)

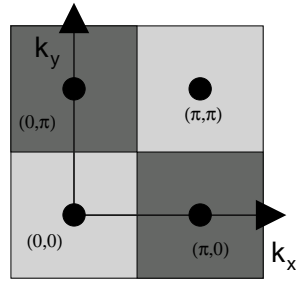

Fig. 1. (a) Overview of the diagrammatic representations of the self-energy used in this work. $\Sigma$ is the electron, and $\Pi$ the phonon self energy. The row labeled ME contains the vertex neglected and VC the corrected diagrams. (b) Schematic of the DCA (quasi-local) formalism for cluster size $N_{C}=4$. Within the squares $\Sigma(\mathbf{k}) \mapsto \Sigma(\mathbf{K})$ where $\mathbf{K}$ are represented by solid circles. Equivalently, $\Delta(\mathbf{k}) \mapsto \Delta(\mathbf{K})$. A partial density of states (DOS) is associated with each sub-zone. DCA enables computations in the thermodynamic limit, via an equivalent cluster impurity problem.

Some treatments of the ARPES kink used a standard Eliashberg theory with anisotropic coupling and an assumed $d$-wave order parameter for fitting purposes $[4,5]$. The extended Eliashberg approach that I have developed differs significantly from previous work in the sense that it is fully self-consistent, with no prior assumptions about the form of the order parameter (i.e. any $d$-wave order arises directly from the self-consistency of the theory) $[6,7]$.

\section{Eliashberg theory and momentum: Failure for optical-phonon mediated $d$-wave states}

In this section, I discuss how quasi-local Eliashberg equations can be constructed, and demonstrate how the lowest-order Fock diagram does not contribute to the gap equations. The calculation is intended to be demonstrative, and shows that standard Eliashberg equations fail for an optical phonon mediated $d$-wave state.

The Eliashberg equations are computed from the Fock contribution to the self-energy:

$$
\begin{aligned}
\hat{\Sigma}\left(\mathbf{k}, i \omega_{n}\right)=-T \sum_{\omega_{n^{\prime}}} \int \frac{d \mathbf{q}}{(2 \pi)^{3}} g_{\mathbf{q}}^{2} \hat{\tau}_{3} \hat{G}\left(\mathbf{k}-\mathbf{q}, \omega_{n^{\prime}}\right) \\
\times \hat{\tau}_{3} K\left(\mathbf{q}, i \omega_{n}-i \omega_{n^{\prime}}\right)
\end{aligned}
$$

and equate $\hat{\Sigma}$ with the approximate form,

$$
\hat{\Sigma} \approx\left(1-Z_{\mathbf{k}}\right) i \omega_{n} \hat{\tau}_{0}+\Delta_{\mathbf{k}}\left(i \omega_{n}\right) \hat{\tau}_{1}+\chi \hat{\tau}_{3}
$$

Normally the Eliashberg equations are computed using a local (momentum-independent) approximation. However, to examine $d$-wave states, the local approximation is not sufficient. Substituting the Green function $\hat{G}\left(\mathbf{k}, i \omega_{n}\right)^{-1}=i \omega_{n} \hat{\tau}_{0}-\zeta_{\mathbf{k}} \hat{\tau}_{3}-\hat{\Sigma}$ in eq. $2\left(\zeta_{\mathbf{k}}=\epsilon_{\mathbf{k}}-\mu, \hat{\tau}\right.$ are Pauli matrices and $\mu$ is the chemical potential), and treating the gap function as constant in sub-zones of $\mathbf{k}$-space in the manner of DCA, one obtains a quasi-local approximation for the Eliashberg equations,

$$
\Delta_{n, \mathbf{K}}=\lambda T \sum_{n^{\prime} \mathbf{Q}} \int d \zeta \frac{\bar{K}\left(n, n^{\prime}\right) \mathcal{D}_{\mathbf{K}-\mathbf{Q}}(\zeta) \Delta_{n^{\prime}, \mathbf{K}-\mathbf{Q}}}{Z_{\mathbf{K}-\mathbf{Q}^{2}}^{2} \omega_{n^{\prime}}^{2}+\zeta^{2}+\Delta_{n^{\prime}, \mathbf{K}-\mathbf{Q}}^{2}}(4)
$$

When gap functions have $d$-wave symmetry, $\Delta_{\pi, 0}=$ $-\Delta_{0, \pi}$ and $\Delta_{0,0}=\Delta_{\pi, \pi}=0$, and the partial DOS $\mathcal{D}(0, \pi)=\mathcal{D}(\pi, 0)$ has the symmetry of the lattice, it is immediately clear that the $\mathcal{O}(\lambda)$ contribution to the quasi-local Eliashberg equations vanishes, and 2nd order terms must be considered. When there is some weak momentum dependence in the e-ph coupling and phonon frequency, this cancellation will not be exact, but the contribution from 2ndorder terms can still be expected to be the largest in the perturbation series. Thus a fully extended set of Eliashberg equations including vertex corrections needs to be constructed. Currently, it is not possible to construct a similar approximation to eq. 4 for the vertex corrected theory. However a numerical approach using the full DCA equations is available to enlighten some aspects of the e-ph problem.

The rest of this article concerns results from an extended Eliashberg theory with momentum dependence and vertex corrections determined numerically using DCA. No form for the order parameter is assumed in advance and full forms for the Green functions and self-energies are used. Full details of the translation of the diagrams in Fig. 1(a) may be found in ref. [6].

\section{Normal state spectroscopy}

Figure 2 shows image plots of the electron spectral functions [8]. Vertex corrections are most pronounced for $\omega_{0}>W \lambda$, as expected from analysis of the parameter space [9]. In particular, the vertex corrected theory shows a kink at the energy scale relating to the phonon frequency measured at the $(\pi, 0)$ point (i.e. renormalised zone edge phonon frequency) in agreement with the ARPES measurements in Ref. [1]. 

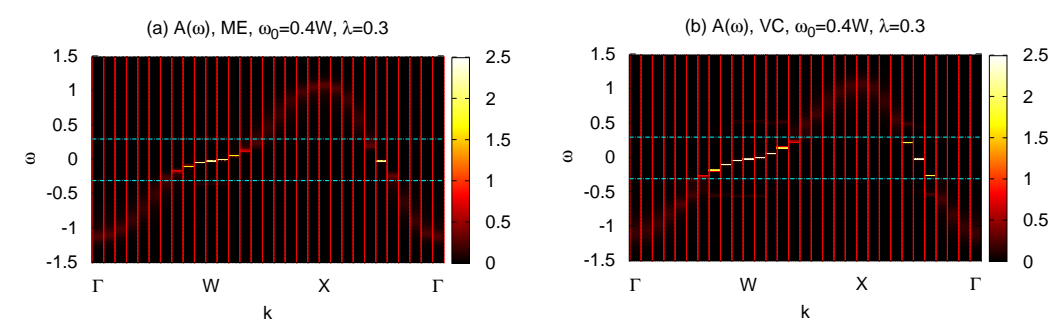

Fig. 2. (Colour online) Electron spectral functions comparing VC and ME approximations when cluster size $N_{C}=64$. The effects of vertex corrections are most pronounced for $\omega_{0}>W \lambda$, as expected from analysis of the parameter space [9]. Horizontal dashed lines indicate the renormalised phonon frequency at the $(\pi, 0)$ zone-edge as computed within the self-consistent theory (i.e. the one which would be measured in a neutron experiment). In particular, only the vertex corrected theory shows a kink at the energy scale relating to the phonon frequency measured at the $(\pi, 0)$ point [8] as reported in Ref. [1].

Phonon spectral functions are shown in Fig. 3. The phonon renormalisation is extremely small for $\omega_{0}=0.4 W$. In contrast, modes at the $(\pi, \pi)$ point in the Brillouin zone are strongly softened for $\omega_{0}=$ $0.2 W$ when vertex corrections are not taken into account. The second order terms act against the softening and reinforce the theory. A small reduction in spectral weight is seen mid way along the $\Gamma$-X and $\Gamma-\mathrm{W}$ lines when $\omega_{0}>W \lambda$. This might be a precursor to the strong softening seen in Ref. [2] on passing through the transition temperature, but further analysis of the superconducting state is necessary to be sure.

\section{Evolution of the order parameter}

$d$-wave superconductivity is one of the remarkable results from the theory extended with vertex corrections. Figure 4 shows the anomalous density as the chemical potential is varied first upwards from half-filling and then back from large filling showing that two solutions are stable. A small $\mu_{c}=1.2$ is applied. $\lambda=0.6$ and $\omega_{0} / W=0.4$. Starting with a half-filled solution and increasing, then decreasing the chemical potential, first $d$-wave and then (on decreasing from high electron density) $s$-wave solutions to the self-consistent equations are found (as detailed in the caption of Fig. 4). Highly anisotropic solutions form as the Fermi-surface approaches the van-Hove points. In the $d$-wave state, the first order terms are not the leading order of the perturbation theory in $\lambda$. It was argued in Ref. [7] that this was in part due to the nearly flat phonon spectrum, so that the phonon propagator $D(\mathbf{k}, \omega) \approx D(\omega)$ and the 1st order contribution to the anomalous self-energy $W \lambda \sum_{\mathbf{q}} D(\mathbf{q}) F(\mathbf{k}-\mathbf{q})$ is small ( $F$ is the anomalous Green function). The anomalous contribution to the pseudopotential term is $\phi_{C}=W \mu_{C} \sum_{\mathbf{k}} F(\mathbf{k})$. Since $F$ is modulated in the $d$-wave state, then the contribution of the pseudopotential term is zero. In the $s$-wave state, $F$ is not modulated, and the resulting finite contribution destroys the $s$-wave order for large enough $\mu_{C}$, thus selecting a dominant $d$-wave contribution. The phonon spectra in Fig. 3 demonstrate that so long as $\omega_{0}>W \lambda$, then this condition is met, and 2nd order terms are essential. 2nd order terms remain the leading order correction to the anomalous self-energy, even for the larger $\lambda$ used here. It is clear that the 2 nd order terms are similar for both repulsive and attractive models, and thus it is perhaps not surprising that both attractive and repulsive models show a $d$-wave state.

\section{Summary and Outlook}

I have described spectroscopic results and order parameters from an extended Eliashberg-style theory with vertex and momentum corrections. The extended theory is essential for the investigation of d-wave superconductivity mediated by optical phonons, since the vertex corrections are the lowest order terms in the perturbation expansion in electron-phonon coupling $\lambda$. Indeed, d-wave superconductivity is immediately predicted with such an extension.

There are two remaining areas which require further investigation. The first involves a better treatment of the Coulomb repulsion, which is currently only treated as a simple Hartree-Fock pseudopotential. In particular, the ability to treat large repulsion and antiferromagnetism is essential for high$T_{C}$ materials. Treatment of momentum dependent e-ph coupling would also be of interest. However, the overriding challenge at this stage is to convert 
(a)

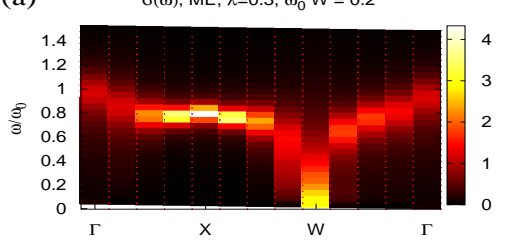

(c)

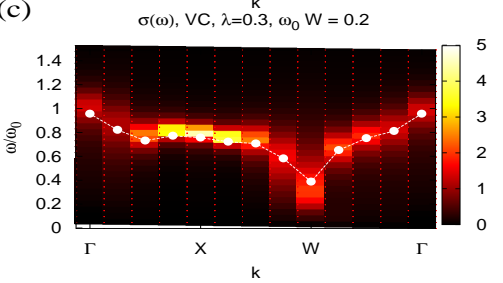

(b)

$\sigma(\omega), M E, \lambda=0.3, \omega_{0} W=0.4$

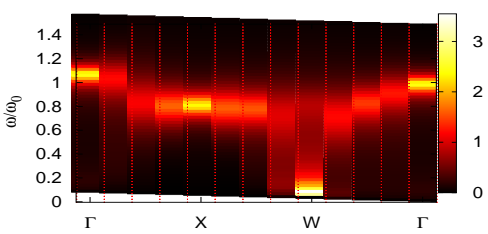

(d)

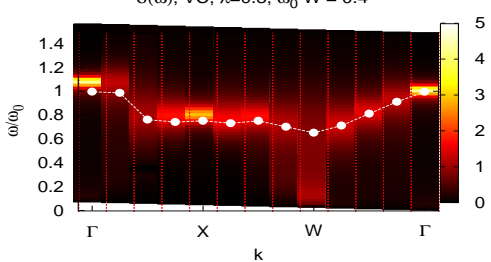

Fig. 3. (Colour online) Phonon spectral functions. The phonon renormalisation is moderate for $\omega_{0}=0.4 W, N_{C}=64$. White points show the values computed from $\omega^{2}-\omega_{0}^{2}(1-\operatorname{Re}[\Pi(\omega)])=0$ for clarification. (a) Modes at the W point in the Brillouin zone are almost completely softened in the theory without vertex corrections (a split mode is indicated in panel (b)). Vertex corrections act against that softening and reinforce the theory $(c, d)$. There is an indication of reduction of spectral weight half way between the high symmetry points when $\omega_{0}>W \lambda$, perhaps with similar origin to that in the neutron results [2]

(a)

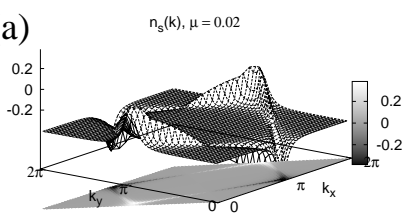

(b)

$n_{s}(k), \mu=0.04$

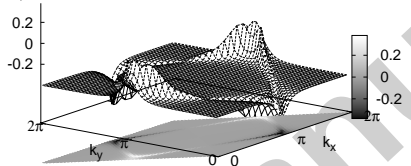

$(\mathrm{c})$

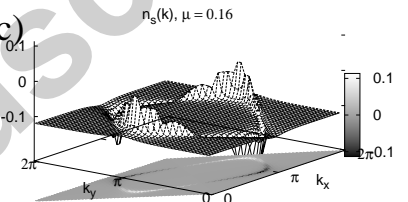

Variation of chemical potential

(f)

(e)

$n_{s}(k), \mu=0.14$

(d)

$n_{\mathbf{s}}(\mathrm{k}), \mu=0.22$
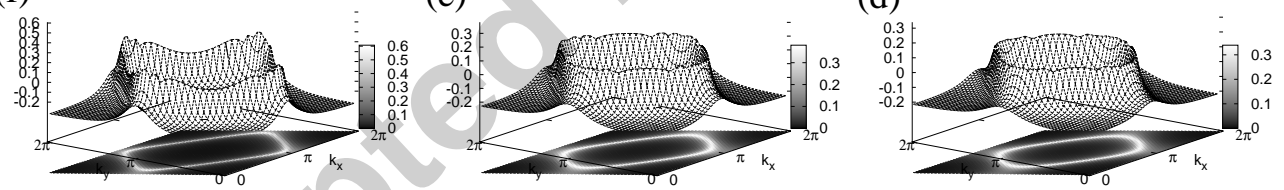

Fig. 4. Anomalous density as $\mu$ is varied first upwards from half-filling and then back from large filling showing two types of solutions to the self-consistent equations. $\mu_{C}=1.2, \lambda=0.6, N_{C}=4$ and $\omega_{0} / W=0.4$. (a) Around half-filling $(n=1)$, there is only one solution with $d$-wave order. This persists to $n=1.175$ where the order parameter spontaneously changes to $s$-wave order $(\mathrm{b}, \mathrm{c})$. The reduction in $d$-wave magnitude is reminiscent of a quantum critical transition. When decreasing $\mu$ from dense filling, the order parameter is a conventional $s$-wave (d), which persists below $n=1.175$, i.e. there are 2 solutions (e). Below $n=1.05$ there is only one solution. Close to $n=1.05$, the $s$-wave solution has an extended-s character, as can be seen in panel (f).

the numerical results into a coherent analytic theory with vertex corrections.

\section{References}

[1] A. Lanzara et. al, Nature 412 (2001) 6846.

[2] J. H. Chung et. al, Phys. Rev. B 67 (2003) 014517.

[3] C. C. Tsuei et. al, Phys. Rev. Lett. 73 (1994) 593.
[4] T. P. Devereaux, T. Cuk, Z.-X. Shen, N. Nagaosa, Phys. Rev. Lett. 93 (2004) 117004.

[5] T. Cuk et. al, Phys. Rev. Lett. 93 (2004) 117003.

[6] J. P. Hague, J. Phys.: Condens. Matter 17 (2005) 5663.

[7] J. P. Hague, Phys. Rev. B 73 (2006) 060503(R).

[8] J. P. Hague, J. Phys.: Condens. Matter 15 (2003) 2535.

[9] J.P.Hague, N.d'Ambrumenil, J. Low Temp. Phys. 140 (2005) 77-89. 